\title{
Exacerbation of bronchiectasis by Pseudomonas putida complicating COVID-19 disease: A case report
}

\author{
VASILIKI EPAMEINONDAS GEORGAKOPOULOU ${ }^{1}$, PANTELIS AVRAMOPOULOS ${ }^{2}$, \\ PETROS PAPALEXIS $^{3,4}$, AIKATERINI BITSANI $^{3}$, CHRISTOS DAMASKOS $^{5,6}$, ANNA GARMPI $^{3}$, \\ AIKATERINI GKOUFA ${ }^{2}$, NIKOLAOS GARMPIS ${ }^{6,7}$, KONSTANTINOS MANTZOURANIS ${ }^{8}$, \\ SERAFEIM CHLAPOUTAKIS ${ }^{9}$, PAGONA SKLAPANI ${ }^{10}$, \\ NIKOLAOS TRAKAS ${ }^{11}$ and DEMETRIOS A. SPANDIDOS ${ }^{12}$
}

\footnotetext{
${ }^{1}$ Pulmonology Department, ${ }^{2}$ First Department of Internal Medicine and ${ }^{3}$ First Department of Propedeutic Internal Medicine, Laiko General Hospital, Medical School, National and Kapodistrian University of Athens, 11527 Athens;

${ }^{4}$ Department of Biomedical Sciences, University of West Attica, 12243 Athens; ${ }^{5}$ Renal Transplantation Unit,

${ }^{6}$ N.S. Christeas Laboratory of Experimental Surgery and Surgical Research and

${ }^{7}$ Second Department of Propedeutic Surgery Laiko General Hospital, Medical School,

National and Kapodistrian University of Athens, 11527 Athens; ${ }^{8}$ st Pulmonology Department Sismanogleio Hospital, 15126 Athens; ${ }^{9}$ Department of Thoracic Surgery, Agios Savvas Hospital, 11522 Athens; ${ }^{10}$ Department of Cytology, Mitera Hospital, 15123 Athens; ${ }^{11}$ Department of Biochemistry, Sismanogleio Hospital, Athens 15126;

${ }^{12}$ Laboratory of Clinical Virology, School of Medicine, University of Crete, 71003 Heraklion, Greece
}

Received September 13, 2021; Accepted October 6, 2021

DOI: $10.3892 / \mathrm{etm} .2021 .10887$

\begin{abstract}
Novel coronavirus infection presents with greater severity in individuals with comorbid chronic lung diseases. Bronchiectasis is an illness characterized by permanent enlargement of the airways, presenting with chronic cough and sputum production and vulnerability to lung infections. Bronchiectasis is not a common comorbid disease in patients with COVID-19 disease and bronchiectasis exacerbation rates were decreased during the pandemic. However, COVID-19 disease is associated with worse outcomes in patients with bronchiectasis and patients with bronchiectasis are more susceptible to SARS-CoV-2 infection development. Pseudomonas putida is an opportunistic pathogen, causing infections mostly in immunocompromised hosts and is not a frequent bacterial colonizer in patients with bronchiectasis. This present study reports a rare case of exacerbation of bronchiectasis by Pseudomonas putida complicating COVID-19 disease in an immunocompetent 70-year-old woman. Clinicians should be aware that SARS-CoV-2 infection is probably a precipitating
\end{abstract}

Correspondence to: Dr Petros Papalexis, First Department of Propedeutic Internal Medicine, Laiko General Hospital, Medical School, National and Kapodistrian University of Athens, 17 Agiou Thoma Street, 11527 Athens, Greece

E-mail: petranpapalex@gmail.com

Key words: bronchiectasis, disease exacerbation, coronavirus, infection, immunocompetence factor of bronchiectasis exacerbation while bronchiectasis is a risk factor for greater severity of SARS-CoV-2 infection.

\section{Introduction}

Bronchiectasis is a chronic pulmonary disease characterized by a vicious cycle of infection and inflammation of the airways, leading to permanent structural lung damage (1). The incidence of the disease has risen since 2000 and there are $\leq 100,000$ individuals in the United States with bronchiectasis, while the disease is also diagnosed more frequently worldwide (2). Bronchiectasis can be detected at all ages and generally presents with chronic cough, sputum production and recurrent lower respiratory tract infections (2). Bronchiectasis is usually post-infection while several lung and systemic diseases are associated with its development (3).

Bronchiectasis exacerbation is a generally infectious clinical condition characterized by deterioration of chronic symptoms with negative consequences on patient prognosis, caused mostly by bacterial microorganisms. Frequent bronchiectasis exacerbations lead to lung damage and rapid decline of lung function (4). Among numerous microorganisms associated with bronchiectasis, Pseudomonas aeruginosa is one of the most frequent and important, with its isolation being associated with frequent exacerbations, poor quality of life and greater possibility of hospitalization and mortality (5).

Novel coronavirus SARS-CoV-2, which causes COVID-19 disease, has exhibited a rapid spread worldwide, becoming the biggest threat ever observed from a pandemic (6). COVID-19 patients have a relatively low rate of bacterial co-infection or bacterial secondary infection. These infections are commonly 
observed in the critically ill patients $(7,8)$. In addition, it has been reported that bronchiectasis exacerbation frequency is significantly decreased during COVID-19 pandemic (9).

Pseudomonas putida is a gram-negative, aerobic saprophytic bacterium that is a common colonizer of soil, plants and water. Due to its remarkable metabolism, it is used as a cell host for synthetic biology and metabolic engineering (10). It has been reported as an opportunistic pathogen causing sepsis in immunocompromised patients and infections among hospitalized patients experiencing invasive procedures due to its ability to contaminate medical solutions $(11,12)$.

The present study reports a novel case of a bronchiectasis exacerbation due to Pseudomonas putida complicating COVID-19 disease.

\section{Case presentation}

A 70-year-old female, non-smoker, patient presented to the Emergency Department of Laiko General Hospital (Athens, Greece) with complaints of fever, runny nose, cough and progressive dyspnea at rest over the previous 10 days. She had a medical history of gastroesophageal reflux, arterial hypertension, hyperlipidemia, asthma, hypothyroidism, obesity with a body mass index (BMI) $33 \mathrm{~kg} / \mathrm{m}^{2}$, obstructive sleep apnea (OSA), pulmonary embolism one year ago and appendectomy. Her medications included pantoprazole, irbesartan/hydrochlorothiazide, atorvastatin, inhaled fluticazone/salmeterol, montelucast, levothyroxine sodium and rivaroxaban. She was also using continuous positive airway pressure therapy for OSA.

Clinical evaluation revealed a febrile patient with wheezing and crackles on auscultation at the bases of both lungs. Clinical examination of the other systems was unremarkable. Blood pressure was $110 / 60 \mathrm{mmHg}$, heart rate was 80 beats per min, oxygen saturation was $90 \%$ on room air and body temperature $38.5^{\circ} \mathrm{C}$. Electrocardiography did not reveal abnormal findings on admission.

Arterial blood gas analysis showed $\mathrm{pO}_{2} 52 \mathrm{mmHg}, \mathrm{pCO}_{2}$ $32 \mathrm{mmHg}$, pH 7.53 and $\mathrm{HCO}_{3} 26.7 \mathrm{mmol} / 1$ on room air. Chest $\mathrm{X}$-Ray showed patchy diffuse infiltrates in both lungs mostly in the periphery of the left lung (Fig. 1A).

Laboratory investigation included complete blood cell count, basic biochemistry serum and urine parameters and coagulation testing. Laboratory findings included hemoglobin $13.1 \mathrm{~g} / \mathrm{dl}$ (normal 12-16 g/dl), hematocrit 39.5\% (normal 38-47\%), white blood cells (WBC) $4.28 \times 10^{3} / \mu 1$ (normal $4-11 \times 10^{3} / \mu 1$ ), neutrophils $2.8 \times 10^{3} / \mu 1$ (normal $1.5-6.6 \times 10^{3} / \mu 1$ ), lymphocytes $1.24 \times 10^{3} / \mu 1$ (normal $1.2-3.4 \times 10^{3} / \mu 1$ ), platelets $145 \times 10^{3} / \mu 1$ (normal $140-440 \times 10^{3} / \mu \mathrm{l}$ ), serumglucose $110 \mathrm{mg} / \mathrm{dl}$ (normal72-106mg/dl), blood urea nitrogen $57 \mathrm{mg} / \mathrm{dl}$ (normal $15-43 \mathrm{mg} / \mathrm{dl}$ ), creatinin $1.36 \mathrm{mg} / \mathrm{dl}$ (normal 0.6-1 mg/dl), C-reactive protein $18.78 \mathrm{mg} / \mathrm{l}$ (normal $<6 \mathrm{mg} / \mathrm{l}$ ), ferritin $145 \mathrm{ng} / \mathrm{ml}$ (normal 15-150 ng/ml), prothrombin time (PT) $28.5 \mathrm{sec}$ (normal 11-13 sec), activated PTT $52.2 \mathrm{sec}$ (normal 29-40 sec), international normalized ratio (INR) 2.16 (normal 0.9-1.2) and d-dimers $1.21 \mu \mathrm{g} / \mathrm{ml}$ (normal $<0.5 \mu \mathrm{g} / \mathrm{ml}$ ). The other blood biochemistry parameters were normal. Urinalysis revealed 1-2 WBCs per high power field (PHF; normal 0-2 PHF), 0-2 RBCs PHF (normal 0-2 PHF), pH 6 (normal 4.5-8), specific gravity 1.020 (normal 1.005-1.025), nitrites negative and leukocyte esterase negative.
The patient was tested for coronavirus and had positive detection of SARS-CoV-2 nucleic acid in examined nasopharyngeal sample using reverse transcription-PCR.

The patient was unvaccinated for SARS-CoV-2. She was transferred to the COVID-19 unit and received oxygen therapy with Venturi mask delivering $35 \%$ oxygen and intravenous dexamethasone, remdesivir and subcutaneous enoxaparin. After three days of hospitalization she presented with resolution of the fever and improvement of oxygen level.

On the fifth day of hospitalization, fever reoccurred and the patient presented with worsening cough with green sputum, with reduction in partial pressure of oxygen, changes in findings from lung auscultation with rhonchi sounds found in all lung fields and worsening infiltrates on chest X-ray (Fig. 1B). The patient received oxygen with Venturi mask delivering $50 \%$ oxygen and intravenous piperacillin-tazobactam empirically. She underwent high resolution computed tomography of the chest showing bronchiectasis and nodular ground glass opacifications mostly with peripheral and subpleural distribution in both lungs (Fig. 2A-C).

Sputum culture was performed which revealed the presence of Pseudomonas putida. Antimicrobial susceptibility testing showed that the microorganism is pansusceptible. The patient presented with improvement after three days of piperacillin-tazobactam administration. She had gradual recovery and she was discharged on the 12nd day of hospitalization without the need of supplemental oxygen and with instructions to receive oral ciprofloxacin in order to have overall a 14-day antibiotic course.

\section{Discussion}

The case is notable for two reasons. First, it is, to the best of the authors' knowledge, the second case in literature mentioning bronchiectasis exacerbation by Pseudomonas putida. The first case was reported by Fujita et al (13) in 1998, in a 57-year-old woman with bronchiectasis and without serious comorbidities. In that case, the patient was non-immunocompromised, had a known history of bronchiectasis, presented with increase in the volume and purulent nature of the sputum and mild hemoptysis and had a right lower lobe consolidation on imaging. The patient in the current study was also an immunocompetent host. She did not present with hemoptysis or consolidation on chest computed tomography scan and she had no known history of bronchiectasis. Pseudomonas putida has emerged as an opportunistic pathogen for non-immunocompetent hosts, causing bacteremia, wound and ocular infections, urinary tract and lower respiratory tract infections, central venous catheter and soft tissue infections in this patients population (14).

Second, the case in the current study indicated that SARS-CoV-2 infection precipitated bronchiectasis exacerbation. Mask-wearing and social distancing during the COVID-19 pandemic were associated with a marked decrease in bronchiectasis exacerbation frequency (15). This decrease was probably noticed due to a reduction in circulating viruses and in traffic-related air pollution, documented during the pandemic (9). However, SARS-CoV-2 infection seems to result in co-infections and exacerbations in patients with bronchiectasis. Lopinto et al (16) reported the first case of acute exacerbation of bronchiectasis, precipitated by COVID-19 

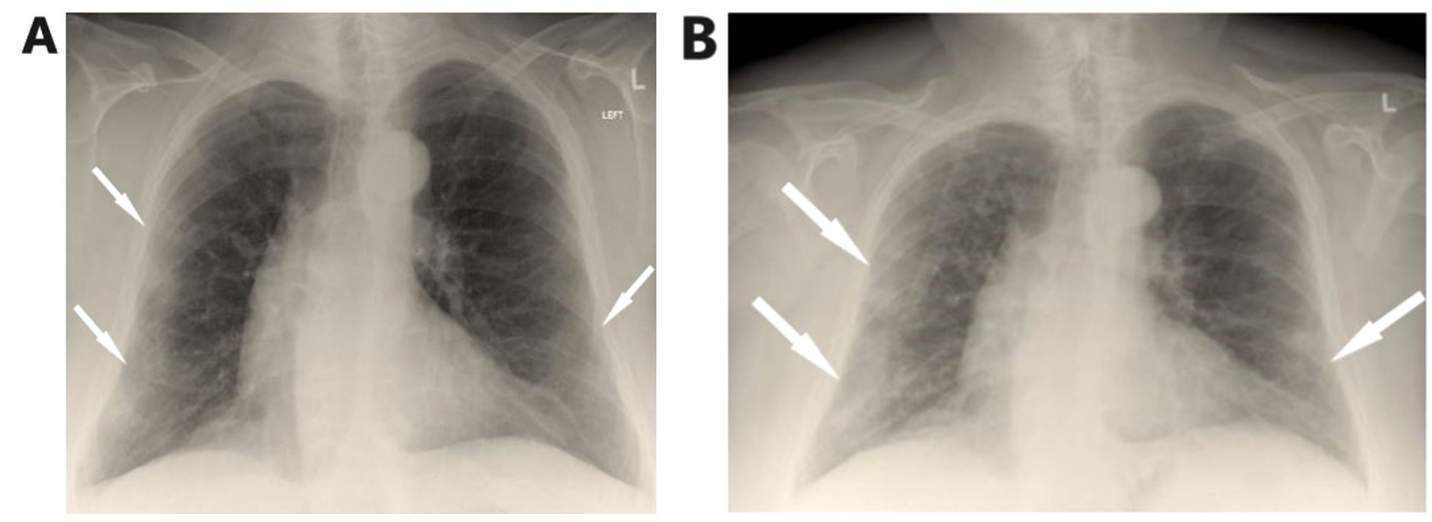

Figure 1. (A) On admission, chest X-ray shows bilateral lung infiltrates, mostly in the periphery of the left lung. (B) Chest X-ray on the 5th day shows worsening of infiltrates.
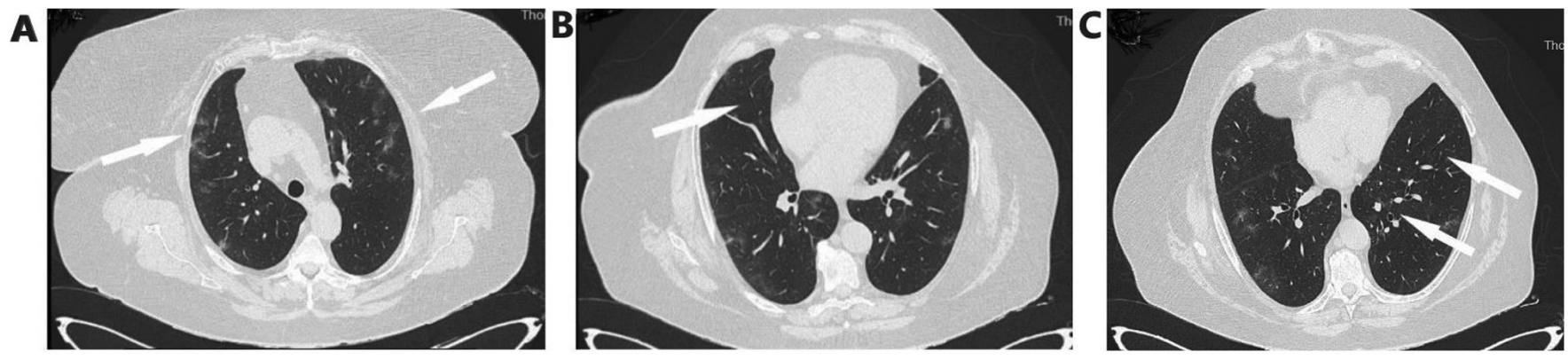

Figure 2. High resolution computed tomography of the chest. (A) Arrows show bilateral pulmonary infiltrates mostly with a peripheral subpleural distribution. (B and C) Arrows show bronchiectasis.

disease and complicated by severe hemoptysis. In addition, Faqihi et al (17) describe a case of co-infection of Bordetella bronchiseptica and novel coronavirus in a young patient with idiopathic bronchiectasis leading to critical illness and requirement of mechanical ventilation. One may hypothesize that the novel virus triggers acute bronchial inflammation and exacerbation of the chronic disease of airways.

It has been reported that $\sim 2 \%$ of patients with COVID-19 have underlying lung disease, which is related to severe clinical manifestations and higher mortality compared with those without chronic lung diseases (18). Bronchiectasis is an uncommon comorbidity observed in COVID-19 patients and thus research about manifestations and clinical outcome of COVID-19 disease in patients with bronchiectasis is limite $(19,20)$. Choi et al $(20)$, in which 8,070 patients with COVID-19 were enrolled, report that the patients with bronchiectasis are significantly older $(\mathrm{P}<0.001)$ and more frequently exhibit pulmonary comorbidities, including asthma. The researchers also reported that the rate of SARS-CoV-2 infection is relatively higher in patients with bronchiectasis compared with those without bronchiectasis and that COVID-19 patients with bronchiectasis present with a higher rate of supplemental oxygen and extracorporeal membrane oxygenation requirement and higher mortality than those without bronchiectasis, indicating that impaired mucociliary clearance and bronchial inflammation possibly increase their vulnerability to, and severity of, COVID-19 (20). Larger prospective studies may be needed to address issues concerning patients with bronchiectasis experiencing SARS-CoV-2 infection.
In conclusion, the present study detailed a rare case of exacerbation of bronchiectasis by Pseudomonas putida complicating COVID-19 disease. Pseudomonas putida can cause pulmonary infection and bronchiectasis exacerbation even in immunocompetent hosts (13). Moreover, SARS-CoV-2 infection should be considered a precipitating factor of bronchiectasis exacerbation, while the presence of bronchiectasis make individuals susceptible to development and greater severity of SARS-CoV-2 infection.

\section{Acknowledgements}

Not applicable.

\section{Funding}

No funding was received.

\section{Availability of data and materials}

All data generated or analyzed during this study are included in this published article.

\section{Authors' contributions}

$\mathrm{PA}, \mathrm{AB}$ and PP conceptualized the case. VEG, KM, NG, CD, AGk and SC wrote and prepared the draft of the manuscript. NT and DAS provided critical revisions. PS and AGa prepared the figures. VEG and AGa confirm the authenticity of all 
the data. All authors contributed to manuscript revision and approved the final version of the manuscript.

\section{Ethics approval and consent to participate}

Not applicable

\section{Patient consent for publication}

Written informed was obtained from the patient for publication of this case report and accompanying images. A copy of the written consent is available for review by the Editor-in-Chief of this journal on request.

\section{Competing interests}

The authors declare that they have no competing interests

\section{References}

1. Cole PJ: Inflammation: A two-edged sword - the model of bronchiectasis. Eur J Respir Dis Suppl 147: 6-15, 1986.

2. O'Donnell AE: Bronchiectasis update. Curr Opin Infect Dis 31: 194-198, 2018

3. Lonni S, Chalmers JD, Goeminne PC, McDonnell MJ, Dimakou K, De Soyza A, Polverino E, Van de Kerkhove C, Rutherford R, Davison J, et al: Etiology of non-cystic fibrosis bronchiectasis in adults and its correlation to disease severity. Ann Am Thorac Soc 12: 1764-1770, 2015.

4. Georgakopoulou VE, Trakas N, Damaskos C, Garmpis N, Karakou E, Chatzikyriakou R, Lambrou P and Tsiafaki X: Neutrophils to lymphocyte ratio as a biomarker in bronchiectasis exacerbation: A retrospective study. Cureus 12: e9728, 2020.

5. Wilson R, Aksamit T, Aliberti S, De Soyza A, Elborn JS, Goeminne P, Hill AT, Menendez R and Polverino E: Challenges in managing Pseudomonas aeruginosa in non-cystic fibrosis bronchiectasis. Respir Med 117: 179-189, 2016.

6. Li X, Yin D, Yang Y, Bi C, Wang Z, Ma G, Fu X, Ji S, Jiang F and Yu T: Eosinophil: A nonnegligible predictor in COVID-19 re-positive patients. Front Immunol 12: 690653, 2021.

7. Lansbury L, Lim B, Baskaran V and Lim WS: Co-infections in people with COVID-19: A systematic review and meta-analysis. J Infect 81: 266-275, 2020.

8. Langford BJ, So M, Raybardhan S, Leung V, Westwood D, MacFadden DR, Soucy JR and Daneman N: Bacterial co-infection and secondary infection in patients with COVID-19: A living rapid review and meta-analysis. Clin Microbiol Infect 26 : 1622-1629, 2020.
9. Crichton ML, Shoemark A and Chalmers JD: The impact of the COVID-19 pandemic on exacerbations and symptoms in bronchiectasis: A prospective study. Am J Respir Crit Care Med 204: 857-859, 2021.

10. Volke DC, Calero P and Nikel PI: Pseudomonas putida. Trends Microbiol 28: 512-513, 2020.

11. Yang CH, Young T, Peng MY and Weng MC: Clinical spectrum of Pseudomonas putida infection. J Formos Med Assoc 95: 754-761, 1996.

12. NeulierC,Breton N,Pangon B,LeMonnier A,Henry-Lagarrigue M, Dujon C and Merrer J: Pseudo-outbreak of Pseudomonas putida respiratory infection caused by laboratory contamination. Infect Control Hosp Epidemiol 32: 523-525, 2011.

13. Fujita J, Negayama K, Ohara M, Hojo S, Obayashi Y, Miyawaki H, Yamaji Y and Takahara J: Pneumonia caused by Pseudomonas putida with a mucoid phenotype. Respir Med 92: 693-695, 1998.

14. Peter S, Oberhettinger P, Schuele L, Dinkelacker A, Vogel W, Dörfel D, Bezdan D, Ossowski S, Marschal M, Liese J, et al: Genomic characterisation of clinical and environmental Pseudomonas putida group strains and determination of their role in the transfer of antimicrobial resistance genes to Pseudomonas aeruginosa. BMC Genomics 18: 859, 2017.

15. Metersky ML: Fewer bronchiectasis exacerbations during the 'Lockdown' for COVID-19: Can we convert knowledge into action? Am J Respir Crit Care Med 204: 759-760, 2021.

16. Lopinto J, Teulier M, Milon A, Voiriot G and Fartoukh M: Severe hemoptysis in post-tuberculosis bronchiectasis precipitated by SARS-CoV-2 infection. BMC Pulm Med 20: 244, 2020.

17. Faqihi F, Alharthy A, Pirompanich P, Noor A, Shahzad A, Nasim N, Balhamar A, Memish ZA and Karakitsos D: Co-infection of SARS-CoV-2 and Bordetella bronchiseptica in a young man with idiopathic non-cystic bronchiectasis and vitamin D3 deficiency. Respir Med Case Rep 31: 101203, 2020.

18. Yang J, Zheng Y, Gou X, Pu K, Chen Z, Guo Q, Ji R, Wang H, Wang Y and Zhou Y: Prevalence of comorbidities and its effects in patients infected with SARS-CoV-2: A systematic review and meta-analysis. Int J Infect Dis 94: 91-95, 2020.

19. $\mathrm{Wu} \mathrm{Z}$ and McGoogan JM: Characteristics of and important lessons from the Coronavirus Disease 2019 (COVID-19) outbreak in China: Summary of a report of 72,314 cases from the Chinese Center for Disease Control and Prevention. JAMA 323: 1239-1242, 2020

20. Choi H, Lee H, Lee SK, Yang B, Chung SJ, Yeo Y, Park TS, Park DW, Moon JY, Kim TH, et al: Impact of bronchiectasis on susceptibility to and severity of COVID-19: A nationwide cohort study. Ther Adv Respir Dis: Feb 14, 2021 (Epub ahead of print). doi: $10.1177 / 1753466621995043$.

(i) $(-)$ This work is licensed under a Creative Commons Attribution-NonCommercial-NoDerivatives 4.0 International (CC BY-NC-ND 4.0) License. 